Bangladesh J. Plant Taxon. 26(2): 269-283, 2019 (December)

(C) 2019 Bangladesh Association of Plant Taxonomists

\title{
ANGIOSPERMIC FLORA OF GAFARGAON UPAZILA OF MYMENSINGH DISTRICT FOCUSING ON MEDICINALLY IMPORTANT SPECIES
}

\author{
M. Oliur Rahman ${ }^{1}$, Nusrat Jahan Sayma and Momtaz Begum \\ Department of Botany, University of Dhaka, Dhaka 1000, Bangladesh
}

Keywords: Angiosperm; Taxonomy; Vegetation analysis; Medicinal Plants; Distribution; Conservation.

\begin{abstract}
Gafargaon upazila has been floristically explored to identify and assess the angiospermic flora that resulted in occurrence of 203 taxa under 174 genera and 75 families. Magnoliopsida is represented by 167 taxa under 140 genera and 62 families, while Liliopsida is constituted by 36 taxa belonging to 34 genera and 13 families. Vegetation analysis shows that herbs are represented by 106 taxa, shrubs 35, trees 54, and climbers by 8 species. In Magnoliopsida, Solanaceae is the largest family possessing 10 species, whereas in Liliopsida, Poaceae is the largest family with 12 species. The study has identified 45 medicinal plants which are used for treatment of over 40 diseases including diabetes, ulcer, diarrhoea, dysentery, fever, cold and cough, menstrual problems, blood pressure and urinary disorders by the local people. Some noticeable medicinal plants used in primary healthcare are Abroma augusta (L.) L.f., Coccinia grandis (L.) Voigt., Commelina benghalensis L., Cynodon dactylon (L.) Pers., Holarrhena antidysenterica Flem., Glycosmis pentaphylla (Retz.) A. DC., Mikania cordata (Burm. f.) Robinson, Ocimum tenuiflorum L. and Rauvolfia serpentina (L.) Benth. A few number of species are also employed in cultural festivals in the study area. Cardamine flexuosa With., Oxystelma secamone (L.) Karst., Phaulopsis imbricata (Forssk.) Sweet, Piper sylvaticum Roxb., Stephania japonica (Thunb.) Miers and Trema orientalis L. have been found to be rare in the investigated area. In order to preserve botanical resources of Gafargaon upazila, particularly the rare, threatened and medicinal plants, conservation measures need to be undertaken through both in-situ and ex-situ methods for their sustainable use.
\end{abstract}

\section{Introduction}

Gafargaon upazila under Mymensingh district is located in $24^{\circ} 15^{\prime}$ to $24^{\circ} 33^{\prime} \mathrm{N}$ and $90^{\circ} 27^{\prime}$ to $90^{\circ} 39^{\prime} \mathrm{E}$ with an area of $401.16 \mathrm{sq} . \mathrm{km}$. The upazila is bounded by Trishal and Nandail upazilas on the north, Kapasia and Sreepur upazilas on the south, Hossainpur and Pakundia upazilas on the east, and Trishal, Bhaluka, and Sreepur upazilas on the west (Fig. 1). The climate of Gafargaon is moderate as other parts of the district, as it is closer to the Himalayas and in the tropical monsoon zone. The temperature of the area varies from $9^{\circ} \mathrm{C}$ to $37^{\circ} \mathrm{C}$. The maximum monthly average humidity ranges from 81 to $97 \%$, while the minimum monthly average humidity ranges from 47 to $79 \%$ illustrates the monthly variations of humidity in the area (BBS, 2018).

The Gafargaon upazila presents diverse habitats and ecosystems comprising wetland, cultivated land, char, homestead area, scrub jungles, fallow lands, etc. which support dense formation of angiosperms and play a pivotal role in the local economy, environment and primary healthcare system. However, the ecosystems of Gafargaon have been depleted due to anthropogenic interferences over the years. As a result, many plant species have become rare and

\footnotetext{
${ }^{1}$ Corresponding author. Email: oliur.bot@du.ac.bd; prof.oliurrahman@gmail.com
} 
threatened. Therefore, it is indispensable to explore, identify, document and preserve the plant wealth of the area for the betterment of mankind especially those plant resources which are used for primary healthcare.
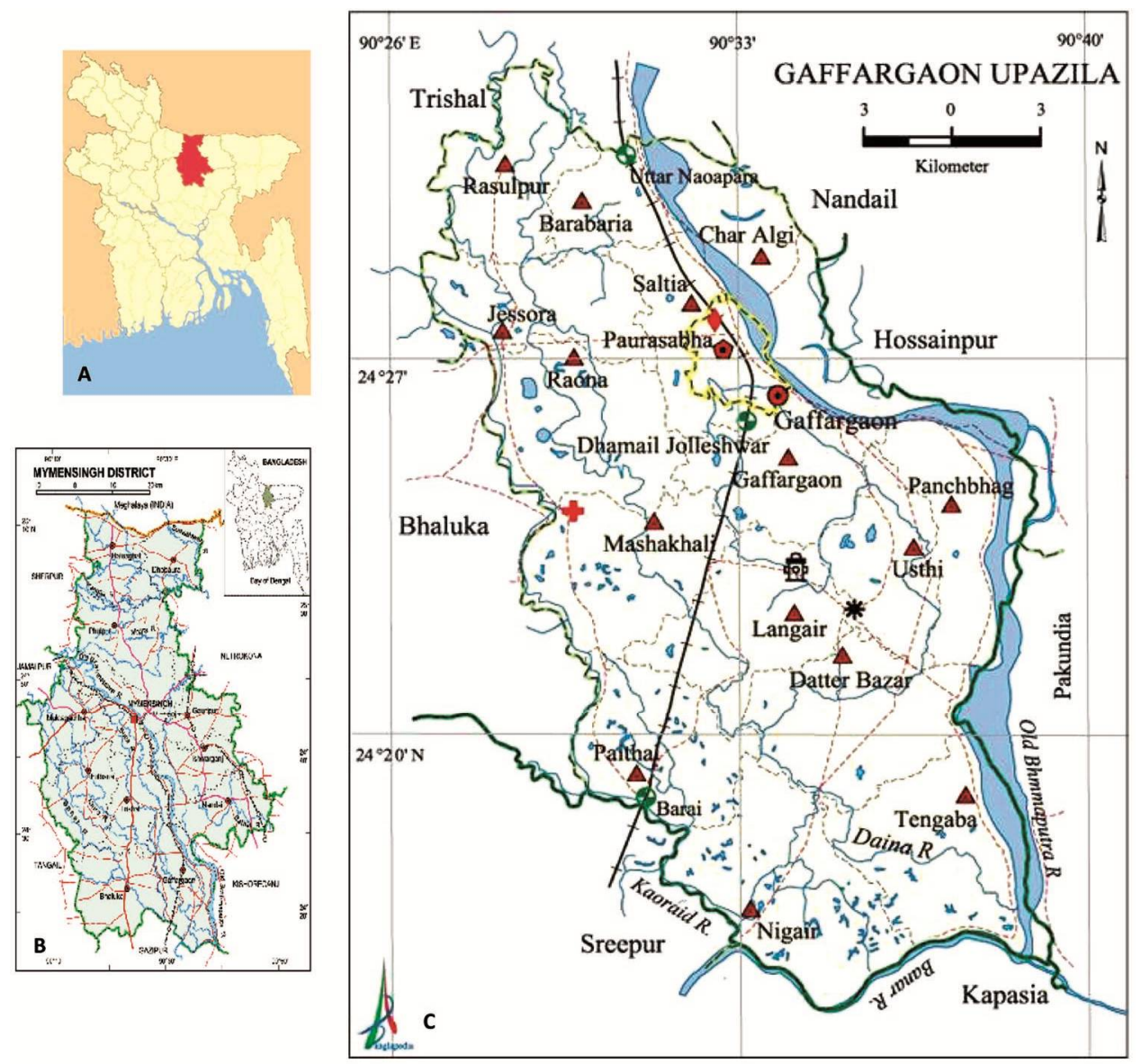

Fig. 1. Map of the study area: A. Map of Bangladesh, B. District map of Mymensingh, C. Map of Gafargaon upazila showing different sampling locations.

In the recent past, several attempts have been made on floristic studies in different parts of the country alongside with some protected areas (Khan and Huq, 2001; Alam et al., 2006; Tutul et al., 2010; Rahman et al., 2015; Arefin et al., 2017; Haque et al., 2018; Rashid et al., 2018). Despite sporadic studies on the flora of some upazilas of the country were carried out earlier (Moniruzzaman et al., 2012; Rahman and Alam, 2013; Rahman et al., 2012, 2013, 2019), the flora of Gafargaon upazila has never been explored and the potential of its existing flora has not been evaluated. Therefore, the present study aimed at exploring and identifying the angiosperm flora of Gafargaon upazila, and to focus on the medicinally important plant resources for meeting up the primary healthcare demand of the local people. The study has the potential to collate primary data on the plant diversity of the upazila which will eventually contribute towards logical understanding and conservation of the biodiversity of this region. 


\section{Materials and Methods}

Taxonomic inventories were conducted in Gafargaon upazila of Mymensingh district through five botanical expeditions covering all seasons from April 2017 to March 2018. Plant specimens with flowers and/or fruits were collected, critically studied and preserved following standard herbarium technique (Bridson and Forman, 1989; Singh and Subramaniam, 2008). The collected specimens were identified by experts, consulting standard literature, viz., Hooker (1872-1897), Prain (1903), Khan (1972-1987), Dassanayake and Fosberg (1980-1985), Khan and Rahman (1989-2002), and by matching with already identified specimens housed at Dhaka University Salar Khan Herbarium (DUSH). Nomenclature of each taxon has been updated following recent literatures (Ahmed et al., 2008-2009), the nomenclatural databases of The Plant List (2013) and TROPICOS (2017). The recognized families are arranged following Cronquist (1981), and the genera and species under each family have been placed in an alphabetical order (Table 1). Bengali name have been cited based on interview with local people, and Huq (1986). Each species is supplemented by its habit, phenology and representative voucher specimen. The information on the uses of medicinal plants has been gathered through interview of the local people. The voucher specimens are preserved at DUSH.

\section{Results and Discussion}

The present study revealed the occurrence of 203 taxa under 174 genera and 75 families in Gafargaon upazila of Mymensingh district. Among them, Magnoliopsida is represented by 62 families, 140 genera and 167 taxa, while Liliopsida (Monocots) is represented by 13 families, 34 genera and 36 taxa. The identified taxa with their Bangla name, family name, habit, status of occurrences and voucher specimen are presented in Table 1.

The present study reveals that largest number of taxa are represented by herbs (106 taxa) followed by trees ( 54 taxa), shrubs ( 35 taxa) and climbers ( 8 taxa). The percentage of identified taxa in Gafargaon upazila is shown in Figure 2. Among the identified taxa $82 \%$ has been found as common and $18 \%$ as rare. In Magnoliopsida, Solanaceae is the largest family comprising 10 species under 7 genera, followed by Fabaceae ( 8 species), and Asteraceae and Amranthaceae (7 species each). In contrast, in Liliopsida, Poaceae is the largest family with 12 species under 10 genera followed by Araceae (6 species) and Arecaceae ( 5 species).

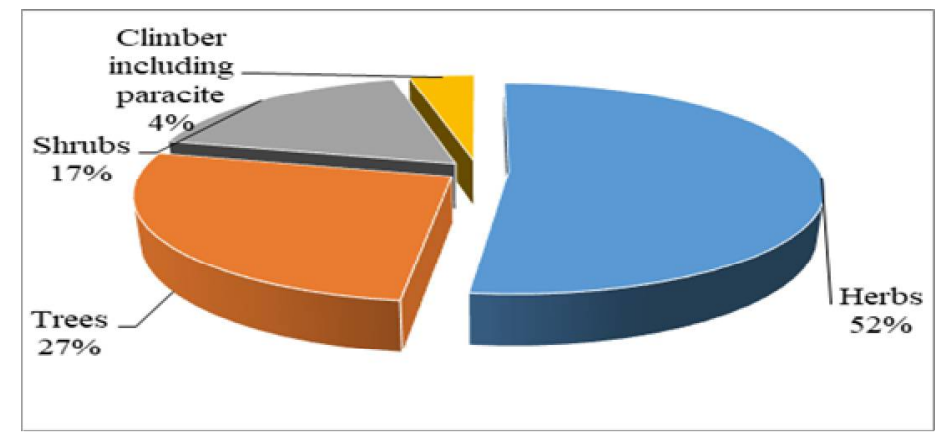

Fig. 2. Pie-chart showing the habitat analysis of identified taxa in Gafargaon upazila.

The families Basellaceae, Bombacaceae, Molluginaceae, Boraginaceae, Bromeliaceae, Capparaceae, Caricaceae, Chenopodiaceae, Commelinaceae, Cuscutaceae, Cyperaceae, Dilleniaceae, Ebenaceae, Elaeocarpaceae, Hydrocharitaceae, Lecythidaceae, Lemnaceae, 
Marantaceae, Melastomataceae, Moringaceae, Musaceae, Oleaceae, Onagraceae, Papaveraceae, Pedaliaceae, Punicaceae, Rhamnaceae, Rosaceae, Salicaceae, Sapindaceae, Sapotaceae, Scrophulariaceae, Sterculiaceae, Tiliaceae, Ulmaceae and Vitaceae are represented by a single species. Ten dominant families of the study area are Poaceae, Solanaceae, Fabaceae, Asteraceae, Amaranthaceae, Caesalpiniaceae, Moraceae, Acanthaceae, Polygonaceae and Nymphaeaceae. The family Poaceae is the largest one represented by 12 species followed by Solanaceae with 10 species and Fabaceae with 8 species (Fig. 3).

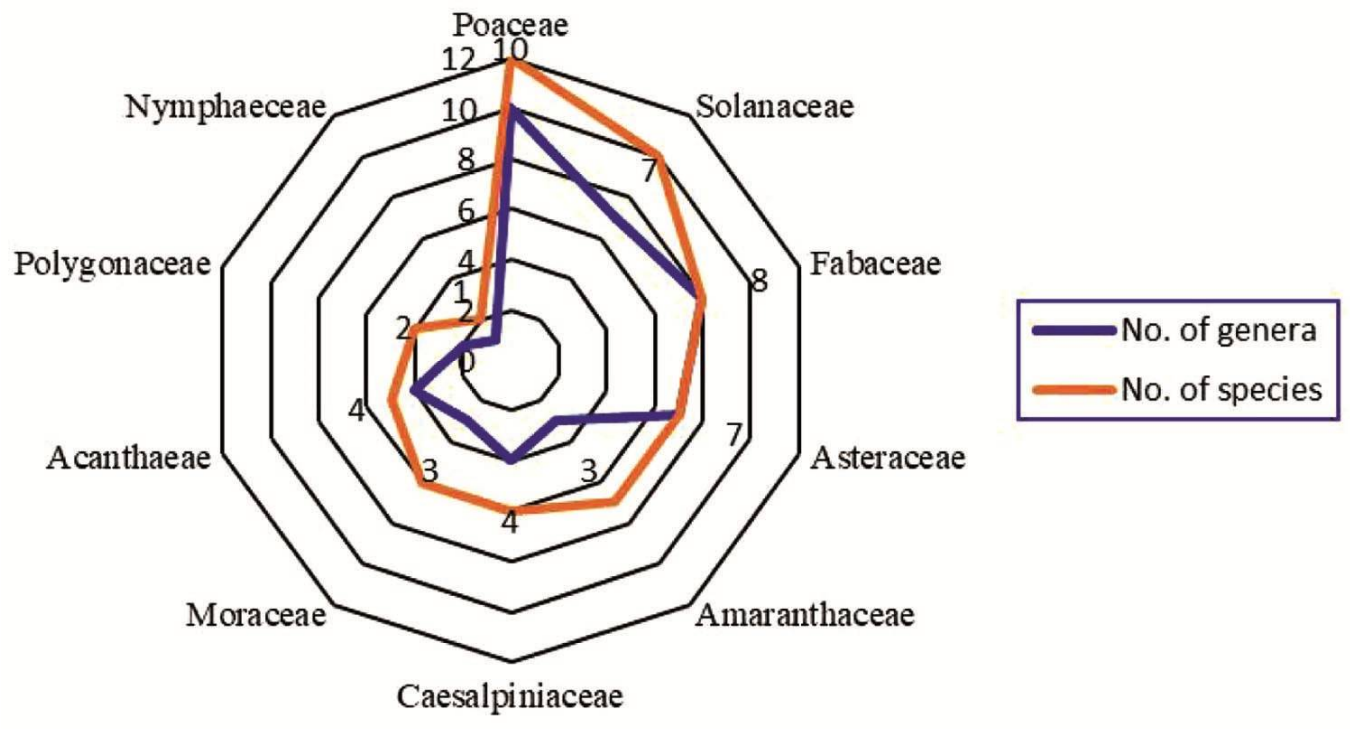

Fig. 3. Radder diagram showing ten dominant plant families of Gafargaon upazila with number of genera and species.

In the study area, some climbers, such as Cissus adnata, Coccinia grandis, Cuscuta reflexa, Stephania japonica etc. grow in homestead trees. Some of the most common trees found in the area include Areca catechu, Albizia lebbeck, Borassus flabellifer, Dalbergia sissoo, Cocos nucifera and Phoenix sylvestris. Commonly growing roadside plants are Phyllanthus reticulatus, Glycosmis pentaphylla, Heliotropium indicum, Solanum nigrum, Croton bonplandianum, Dalbergia sissoo, Senna tora etc. Most common homestead plants are Hibiscus rosa-sinensis, Litchi chinensis, Artocarpus heterophyllus, Psidium guajava, Lawsonia inermis, Averrhoa carambola, Swietenia mahagoni and Punica granatum. In the investigated area Cardamine flexuosa, Oxystelma secamone, Phaulopsis imbricata, Piper sylvaticum, Stephania japonica and Trema orientalis have been found as rare based on field observation. Gafargaon upazila is also endowed with different aquatic habitats including beels, ponds, tanks and other low-lying areas with seasonal water. Some of the important aquatic angiosperms are Pistia stratiotes, Oxystelma secamone, Enhydra fluctuans, Ipomoea fistulosa, Ipomoea aquatica, Ottelia alismoides, Nymphaea pubescens, Nymphaea rubra, Lemna perpusilla, Ludwigia adscendens and Monochoria hastata. 
Table 1. Plant species of Gafargaon upazila of Mymensigh district with their Bengali names, habit, phenology, status of occurrence and vouchers.

\begin{tabular}{|c|c|c|c|c|c|}
\hline Taxa & Bengali name & Habit & Phenology & $\begin{array}{l}\text { Status of } \\
\text { occurrence }\end{array}$ & $\begin{array}{l}\text { Voucher } \\
\text { number }\end{array}$ \\
\hline \multicolumn{6}{|l|}{ MAGNOLIOPSIDA } \\
\hline \multicolumn{6}{|l|}{ Annonaceae } \\
\hline Annona reticulata $\mathrm{L}$. & Ata & Tree & Oct-Jan & Common & Nusrat 01 \\
\hline A. squamosa $\mathrm{L}$. & Sharifa & Tree & Mar-Dec & Common & Nusrat 57 \\
\hline Polyalthia longifolia (Sonn.) Thw. & Debdaru & Tree & Mar-Sep & Common & Nusrat 02 \\
\hline Uvaria hamiltonii Hook. f. & Latkan & Shrub & May-Oct & Common & Nusrat 65 \\
\hline \multicolumn{6}{|l|}{ Lauraceae } \\
\hline Cinnamomum camphora Prain & Karpur & Tree & Mar-Jul & Common & Nusrat 28 \\
\hline C. tamala Nees \& Eberm. & Tejpata & Tree & Feb-Oct & Common & Nusrat 182 \\
\hline Litsea glutinosa (Lour.) Robinson & Menda & Tree & Apr-Jan & Common & Nusrat 85 \\
\hline \multicolumn{6}{|l|}{ Piperaceae } \\
\hline Peperomia pellucida (L.) Kunth & Luchipata & Herb & Jul-Sep & Common & Nusrat 191 \\
\hline Piper nigrum $\mathrm{L}$. & Goolmorich & Climber & Aug-Dec & Common & Nusrat 97 \\
\hline P. sylvaticum Roxb. & Bon pan & Shrub & & Rare & Nusrat 192 \\
\hline \multicolumn{6}{|l|}{ Nymphaeaceae } \\
\hline Nymphaea pubescens Willd. & Saluk & Herb & Jan-Dec & Common & Nusrat 188 \\
\hline Nymphaea rubra Roxb. ex Salisb. & Lalsapla & Herb & Jul-Jan & Common & Nusrat 95 \\
\hline \multicolumn{6}{|l|}{ Menispermaceae } \\
\hline Stephania japonica (Thunb.) Miers & Doipata & Climber & Jan-Dec & Rare & Nusrat 142 \\
\hline Tinospora crispa (T.) Hook. f. & Gulonchoe & Climber & Jan-Jun & Common & Nusrat 90 \\
\hline \multicolumn{6}{|l|}{ Papaveraceae } \\
\hline Argemone mexicana $\mathrm{L}$. & Sialkanta & Herb & Feb-Jun & Common & Nusrat 190 \\
\hline Ulmaceae & & & & & Nusrat \\
\hline Trema orientalis $\mathrm{L}$. & Jinal & Tree & Dec-Apr & Rare & Nusrat 55 \\
\hline \multicolumn{6}{|l|}{ Moraceae } \\
\hline Artocarpus heterophyllus Lamk. & Kanthal & Tree & Feb-Jun & Common & Nusrat 92 \\
\hline A. laucha Buch.-Ham. & Dewa & Tree & Apr-Aug & Common & Nusrat 145 \\
\hline Ficus benghalensis $\mathrm{L}$. & Bot & Tree & May-Aug & Common & Nusrat 38 \\
\hline F. hispida L. f. & Dumur & Shrub & Apr-Sep & Common & Nusrat 186 \\
\hline F. religiosa $\mathrm{L}$. & Ashwatha & Tree & Mar-Sep & Common & Nusrat 37 \\
\hline Sreblus asper Lour. & Sheora & Tree & Feb-Jun & Common & Nusrat 160 \\
\hline \multicolumn{6}{|l|}{ Urticaceae } \\
\hline Laportea crenulata Gaud. & Churapata & Shrub & May-Sep & Common & Nusrat 112 \\
\hline L. interrupta $\mathrm{L}$. & Bichuti & Herb & Aug-Nov & Rare & Nusrat 53 \\
\hline \multicolumn{6}{|l|}{ Nyctaginaceae } \\
\hline Boerhavia diffusa $\mathrm{L}$. & Punarnava & Herb & Apr-Aug & Common & Nusrat 39 \\
\hline Bougainvillea spectabilis Willd. & Baganbilash & Shrub & Jan-Dec & Common & Nusrat 94 \\
\hline Mirabilis jalapa $\mathrm{L}$. & Shandhamalati & Herb & Mar-May & Common & Nusrat 147 \\
\hline \multicolumn{6}{|l|}{ Chenopodiaceae } \\
\hline Chenopodium album $\mathrm{L}$. & Bathua-shak & Herb & Dec-Mar & Common & Nusrat 20 \\
\hline
\end{tabular}


Table 1 contd.

\begin{tabular}{|c|c|c|c|c|c|}
\hline Taxa & Bengali name & Habit & Phenology & $\begin{array}{l}\text { Status of } \\
\text { occurrence }\end{array}$ & $\begin{array}{l}\text { Voucher } \\
\text { number }\end{array}$ \\
\hline \multicolumn{6}{|l|}{ Amaranthaceae } \\
\hline Achyranthus aspera $\mathrm{L}$. & Apang & Herb & Jan-Dec & Rare & Nusrat 117 \\
\hline $\begin{array}{l}\text { Alternenthera philoxeroides (Mart) } \\
\text { Griseb. }\end{array}$ & \multicolumn{4}{|c|}{ Griseb. } & Nusrat 163 \\
\hline A. sessilis (L.) DC. & Sachi-shak & Herb & Jan-Dec & Common & Nusrat 59 \\
\hline Amaranthus blitum $\mathrm{L}$. & Natiyashak & Herb & Oct-Nov & Common & Nusrat 118 \\
\hline A. spinosus $\mathrm{L}$. & Katanotay & Herb & Jan-Dec & Common & Nusrat 05 \\
\hline A. tricolor $\mathrm{L}$. & Denga & Herb & Jan-Dec & Common & Nusrat 164 \\
\hline A. viridis $\mathrm{L}$. & Notay-shak & Herb & Jan-Dec & Common & Nusrat 60 \\
\hline \multicolumn{6}{|l|}{ Basellaceae } \\
\hline Basella rubra $\mathrm{L}$. & Puishak & Herb & Nov-Mar & Common & Nusrat 173 \\
\hline \multicolumn{6}{|l|}{ Molluginaceae } \\
\hline Glinus oppositifolius (L.) A. DC. & Ghema shak & Herb & Jan-Dec & Common & Nusrat 203 \\
\hline \multicolumn{6}{|l|}{ Polygonaceae } \\
\hline $\begin{array}{l}\text { Persicaria flaccida (Meissn.) } \\
\text { H. Gross ex Loeseeen }\end{array}$ & Lal bishkatali & Herb & Apr-Aug & Common & Nusrat 152 \\
\hline P. hydropiper (L.) Spach. & Biskatali & Herb & Apr-Aug & Common & Nusrat 101 \\
\hline $\begin{array}{l}\text { P. stagnina (Buch-Ham. ex Meissn.) } \\
\text { M.A. Hassan }\end{array}$ & Bara bishkatali & Herb & Apr-Dec & Common & Nusrat 102 \\
\hline Rumex dentatus $\mathrm{L}$. & Daton & Herb & Jan-May & Common & Nusrat 196 \\
\hline \multicolumn{6}{|l|}{ Dilleniaceae } \\
\hline Dillenia indica $\mathrm{L}$. & Chalta & Tree & May-Feb & Common & Nusrat 23 \\
\hline \multicolumn{6}{|l|}{ Elaeocarpaceae } \\
\hline Elaeocarpus floribundus B1. & Jalpai & Tree & Mar-Dec & Common & Nusrat 77 \\
\hline \multicolumn{6}{|l|}{ Tiliaceae } \\
\hline Corchorus capsularis L. & Deshipat & Herb & Jun-Nov & Common & Nusrat 111 \\
\hline \multicolumn{6}{|l|}{ Sterculiaceae } \\
\hline Abroma augusta (L.) L. f. & Ulatkombal & Shrub & Jun-Dec & Rare & Nusrat 159 \\
\hline \multicolumn{6}{|l|}{ Bombacaceae } \\
\hline Bombax ceiba $\mathrm{L}$. & Shimultula & Tree & Jan-Apr & Common & Nusrat 127 \\
\hline \multicolumn{6}{|l|}{ Malvaceae } \\
\hline Abelmoschus escunlentus (L.) Moench & Dheros & Herb & Jan-Dec & Common & Nusrat 87 \\
\hline Abutilon indicum $\mathrm{L}$. & Petari & Herb & Jul-Apr & Common & Nusrat 140 \\
\hline Hibiscus rosa-sinensis $\mathrm{L}$. & Joba & Shrub & Jan-Dec & Common & Nusrat 202 \\
\hline Sida cordata (Burm.f.) Borss. & Junka & Herb & Aug-Feb & Common & Nusrat 184 \\
\hline Urena lobata $\mathrm{L}$. & Bon okra & Shrub & Jan-Dec & Common & Nusrat 31 \\
\hline \multicolumn{6}{|l|}{ Lecythidaceae } \\
\hline Barringtonia acutangula (L.) Gaertn. & Hijal & Tree & May-Sep & Common & Nusrat 138 \\
\hline \multicolumn{6}{|l|}{ Caricaceae } \\
\hline Carica papaya $\mathrm{L}$. & Pape & Herb & Jan-Dec & Common & Nusrat 19 \\
\hline \multicolumn{6}{|l|}{ Cucurbitaceae } \\
\hline Benincasa hispida (Thunb.) Cogn. & Chalkumra & Climber & May-Nov & Common & Nusrat 21 \\
\hline Coccinia grandis (L.) Voigt. & Telakucha & Climber & Mar-Dec & Common & Nusrat 132 \\
\hline
\end{tabular}


Table 1contd.

\begin{tabular}{|c|c|c|c|c|c|}
\hline Taxa & Bengali name & Habit & Phenology & $\begin{array}{l}\text { Status of } \\
\text { occurrence }\end{array}$ & $\begin{array}{l}\text { Voucher } \\
\text { number }\end{array}$ \\
\hline Cucumis melo $\mathrm{L}$. & Baangi & Climber & Mar-Oct & Rare & Nusrat 177 \\
\hline C. sativus $\mathrm{L}$. & Khira & Herb & Apr-Oct & Common & Nusrat 74 \\
\hline Cucurbita maxima Duch. ex Lamk. & Mistikumra & Herb & Apr-Oct & Common & Nusrat 75 \\
\hline \multicolumn{6}{|l|}{ Salicaceae } \\
\hline Salix tetrasperma Roxb & Panihijal & Tree & Nov-Mar & Rare & Nusrat 51 \\
\hline \multicolumn{6}{|l|}{ Capparaceae } \\
\hline Crateva magna (Lour.) DC. & Barun & Tree & Feb-May & Common & Nusrat 130 \\
\hline \multicolumn{6}{|l|}{ Brasicaceae } \\
\hline Cardamine flexuosa With. & Not known & Herb & Feb-Jul & Rare & Nusrat 173 \\
\hline Brassica napus L. & Sorisha & Herb & Mar-Jul & Common & Nusrat 128 \\
\hline Raphanus sativus L. & Mula & Herb & Jan-May & Common & Nusrat 16 \\
\hline Rorippa indica (L.) Hiern & Bansarisa & Herb & Apr-Jan & Common & Nusrat 69 \\
\hline \multicolumn{6}{|l|}{ Moringaceae } \\
\hline Moringa oleifera Lamk. & Sajna & Tree & Oct-Mar & Common & Nusrat 40 \\
\hline \multicolumn{6}{|l|}{ Sapotaceae } \\
\hline Manilkara zapota (L.) P.Van Royen & Sofeda & Tree & Jan-Dec & Common & Nusrat 156 \\
\hline \multicolumn{6}{|l|}{ Ebenaceae } \\
\hline Diospyros malabarica (Desr.) Kostel & Deshi gab & Tree & May-Aug & Rare & Nusrat 133 \\
\hline \multicolumn{6}{|l|}{ Rosaceae } \\
\hline Rosa chinensis Jacq. & Golap & Shrub & Nov-Mar & Common & Nusrat 47 \\
\hline \multicolumn{6}{|l|}{ Mimosaceae } \\
\hline $\begin{array}{l}\text { Acacia auriculiformis A. Cunn. ex } \\
\text { Benth. }\end{array}$ & Akashmoni & Tree & Jun-Feb & Common & Nusrat 185 \\
\hline A. nilotica (L.) Willd. ex Del. & Babla & Tree & Aug-May & Common & Nusrat 143 \\
\hline Albizia lebbeck L. & Koroi & Tree & Apr-Oct & Common & Nusrat 35 \\
\hline A. procera (Roxb.) Benth & SilKoroi & Tree & Jun-Nov & Common & Nusrat 91 \\
\hline $\begin{array}{l}\text { Leucaena leucocephala (Lam.) De } \\
\text { Wit. }\end{array}$ & Ipl-ipl & Tree & Mar-Nov & Common & Nusrat 204 \\
\hline Mimosa pudica $\mathrm{L}$. & Lajjaboti & Herb & Sep-Dec & Common & Nusrat \\
\hline \multicolumn{6}{|l|}{ Caesalpinaceae } \\
\hline Cassia fistula $\mathrm{L}$. & Banarlathi & Tree & Mar-Jun & Common & Nusrat 175 \\
\hline Delonix regia Rafin. & Krisnochura & Tree & Apr-Sep & Common & Nusrat 70 \\
\hline Senna occidentalis Roxb. & Borakalkasuna & Herb & May-Oct & Common & Nusrat 129 \\
\hline S. sophera (L.) Roxb. & Kalkashunda & Shrub & Sep-Jul & Common & Nusrat 174 \\
\hline S. tora (L.) Roxb. & Chakunda & Herb & Jul-Dec & Common & Nusrat 18 \\
\hline Tamarindus indica $\mathrm{L}$. & Tentul & Tree & Apr-Dec & Common & Nusrat 205 \\
\hline \multicolumn{6}{|l|}{ Fabaceae } \\
\hline Arachis hypogaea $\mathrm{L}$. & Badam & Herb & Mar-Dec & Common & Nusrat 25 \\
\hline Cajanus cajan (L.) Millsp. & Orhor & Shrub & Dec-Apr & Common & Nusrat 79 \\
\hline Crotalaria pallida Ait. & Jhun-jhuni & Herb & May-Dec & Rare & Nusrat 180 \\
\hline Dalbergia sissoo Roxb. & Sisso & Tree & Mar-Jun & Common & Nusrat 135 \\
\hline $\begin{array}{l}\text { Desmodium heterophyllum (Willd.) } \\
\text { DC. }\end{array}$ & Kodalia & Herb & Jan-Dec & Rare & Nusrat 26 \\
\hline
\end{tabular}


Table 1 contd.

\begin{tabular}{|c|c|c|c|c|c|}
\hline Taxa & Bengali name & Habit & Phenology & $\begin{array}{l}\text { Status of } \\
\text { occurrence }\end{array}$ & $\begin{array}{l}\text { Voucher } \\
\text { number }\end{array}$ \\
\hline Lablab purpureus (L.) Sweet & Shim & Herb & Nov-Mar & Common & Nusrat 80 \\
\hline Sesbania grandiflora $\mathrm{L}$. & Bakful & Tree & Oct-Feb & Common & Nusrat 179 \\
\hline Vigna mungo (L.) Hepper & Mashkalai & Herb & Nov-Jan & Common & Nusrat 136 \\
\hline \multicolumn{6}{|l|}{ Lythraceae } \\
\hline Lawsonia inermis $\mathrm{L}$. & Mahendi & Shrub & Jun-Dec & Common & Nusrat 30 \\
\hline \multicolumn{6}{|l|}{ Myrtaceae } \\
\hline Psidium guajava $\mathrm{L}$. & Payara & Tree & Apr-Sep & Common & Nusrat 187 \\
\hline Syzygium cumini L. & Kalojam & Tree & Apr-Jul & Common & Nusrat 93 \\
\hline \multicolumn{6}{|l|}{ Punicaceae } \\
\hline Punica granatum $\mathrm{L}$. & Dalim & Shrub & Jan-Dec & Common & Nusrat 197 \\
\hline \multicolumn{6}{|l|}{ Onagraceae } \\
\hline Ludwigia adscendens (L.) Hara & Kesardam & Herb & Mar-Dec & Common & Nusrat 42 \\
\hline \multicolumn{6}{|l|}{ Melastomataceae } \\
\hline Melastoma malabathricum $\mathrm{L}$. & Ban tezpata & Shrub & Jan-Dec & Rare & Nusrat 88 \\
\hline \multicolumn{6}{|l|}{ Euphorbiaceae } \\
\hline Baccaurea ramiflora Lour. & Lotkon/Bobi & Tree & Jun-Sep & Common & Nusrat 24 \\
\hline Croton bonplandianus Baill. & Croton & Herb & Jan-Dec & Common & Nusrat 178 \\
\hline Phyllanthus reticulatus Poir. & Chitki & Shrub & Mar-Oct & Common & Nusrat 134 \\
\hline Ricinus communis $\mathrm{L}$. & Verenda & Shrub & Jan-Dec & Rare & Nusrat 78 \\
\hline \multicolumn{6}{|l|}{ Rhamnaceae } \\
\hline Ziziphus mauritiana Lamk. & Boroi & Tree & Sep-Mar & Common & Nusrat 153 \\
\hline \multicolumn{6}{|l|}{ Vitaceae } \\
\hline Cissus adnata Roxb. & Alingolata & Climber & Mar-Aug & Common & Nusrat 115 \\
\hline \multicolumn{6}{|l|}{ Sapindaceae } \\
\hline Litchi chinensis Sonn. & Lichu & Tree & Apr-Jun & Common & Nusrat 107 \\
\hline \multicolumn{6}{|l|}{ Anacardiaceae } \\
\hline Mangifera indica $\mathrm{L}$. & Aam & Tree & Jan-Jun & Common & Nusrat 06 \\
\hline Spondias pinnata (L.f.) Kurz & Amra & Tree & Feb-Aug & Common & Nusrat 119 \\
\hline \multicolumn{6}{|l|}{ Meliaceae } \\
\hline $\begin{array}{l}\text { Aphanamixis polystachya (Wall.) R.N. } \\
\text { Parker }\end{array}$ & Baiddiraj & Tree & Feb-May & Rare & Nusrat 33 \\
\hline Azadirachta indica A. Juss. & Neem & Tree & Mar-Jul & Common & Nusrat 89 \\
\hline Melia azedarach $\mathrm{L}$. & Ghoranim & Tree & Mar-Feb & Common & Nusrat 141 \\
\hline Swietenia mahagoni Jacq. & Mahagoni & Tree & Apr-Nov & Common & Nusrat 34 \\
\hline \multicolumn{6}{|l|}{ Rutaceae } \\
\hline Aegle marmelos (L.) Correa & Bel & Tree & Apr-Dec & Common & Nusrat 48 \\
\hline $\begin{array}{l}\text { Citrus aurantifolia (Christm. \& } \\
\text { Panzer) Swingle }\end{array}$ & Lebu & Shrub & Mar-Sep & Common & Nusrat 105 \\
\hline Glycosmis pentaphylla (Retz.) A.DC. & Motkila & Shrub & Jan-Dec & Common & Nusrat 155 \\
\hline Zanthoxylum rhetsa (Roxb.) DC. & Bajna & Tree & Mar-Sep & Common & Nusrat 106 \\
\hline \multicolumn{6}{|l|}{ Oxalidaceae } \\
\hline Averrhoa carambola $\mathrm{L}$. & Kamranga & Tree & Sep-Mar & Common & Nusrat 96 \\
\hline Oxalis corniculata $\mathrm{L}$. & Amrul & Herb & Sep-May & Common & Nusrat 108 \\
\hline
\end{tabular}


Table 1 contd.

\begin{tabular}{|c|c|c|c|c|c|}
\hline Taxa & Bengali name & Habit & Phenology & $\begin{array}{l}\text { Status of } \\
\text { occurrence }\end{array}$ & $\begin{array}{l}\text { Voucher } \\
\text { number }\end{array}$ \\
\hline \multicolumn{6}{|l|}{ Apiaceae } \\
\hline Centella asiatica (L.) Urban & Thankuni & Herb & Apr-Dec & Rare & Nusrat 61 \\
\hline Coriandrum savitum $\mathrm{L}$. & Dhony & Herb & Dec-Feb & Common & Nusrat 165 \\
\hline Foeniculum vulgare Mill. & Pan-mouri & Herb & Nov-Feb & Common & Nusrat 07 \\
\hline \multicolumn{6}{|l|}{ Apocynaceae } \\
\hline Alstonia scholaris (L.) R.Br. & Chatim & Tree & Oct-Jun & Rare & Nusrat 166 \\
\hline Carissa carandus $\mathrm{L}$. & Karamcha & Shrub & Apr-Oct & Common & Nusrat 167 \\
\hline Catharanthus roseus (L.) G. Don & Noyontara & Herb & Jan-Dec & Common & Nusrat 127 \\
\hline Holarrhena antidysenterica Flem. & Kurchi & Shrub & Apr-Dec & Common & Nusrat 08 \\
\hline Rauvolfia serpentina (L.) Benth. & Sarpagandha & Herb & Apr-Oct & Rare & Nusrat 62 \\
\hline $\begin{array}{l}\text { Tabernaemontana divaricata (L.) R. } \\
\text { Br. ex Roem. \& Schult. }\end{array}$ & Togor & Shrub & May-Jan & Common & Nusrat 121 \\
\hline \multicolumn{6}{|l|}{ Asclepediaceae } \\
\hline Calotropis gigantea $\mathrm{L}$. & Akondo & Shrub & Jan-Dec & Rare & Nusrat 124 \\
\hline Oxystelma secamone (L.) Karst. & Dudhialata & Herb & Aug-Oct & Rare & Nusrat 12 \\
\hline \multicolumn{6}{|l|}{ Solanaceae } \\
\hline Capsicum frutescens $\mathrm{L}$. & Kacha-morich & Herb & Jan-Dec & Common & Nusrat 108 \\
\hline Cestrum nocturnum $\mathrm{L}$. & Hasnahena & Shrub & Jan-Dec & Common & Nusrat \\
\hline Datura metel $\mathrm{L}$. & Datura & Shrub & Jan-Dec & Rare & Nusrat 201 \\
\hline Lycopersicon esculentum Mill. & Tomato & Herb & Oct-Apr & Common & Nusrat 50 \\
\hline Nicotiana plumbaginifolia Willd. & Ban-tamak & Herb & Mar-Dec & Common & Nusrat 109 \\
\hline Physalis angulata $\mathrm{L}$. & Fotka & Herb & Feb-Aug & Common & Nusrat 52 \\
\hline P. minima $\mathrm{L}$. & Chotofotka & Herb & Jan-Dec & Common & Nusrat 202 \\
\hline Solanum melongena $\mathrm{L}$. & Begun & Herb & Oct-Mar & Common & Nusrat 158 \\
\hline S. torvum Swartz. & Gotabegun & Shrub & Jan-Dec & Common & Nusrat 110 \\
\hline S. tuberosum L. & Gol-alu & Herb & Jan-Mar & Common & Nusrat 54 \\
\hline \multicolumn{6}{|l|}{ Convolvulaceae } \\
\hline Ipomoea aquatica Forssk. & Kolmishak & Herb & Jan-Dec & Common & Nusrat 176 \\
\hline I. batatas (L.) Lamk. & Misti-alu & Herb & Dec-May & Rare & Nusrat 73 \\
\hline I. fistulosa Mart. ex Choisy & Dholkolmi & Shrub & Jan-Dec & Common & Nusrat 131 \\
\hline \multicolumn{6}{|l|}{ Cuscutaceae } \\
\hline Cuscuta reflexa Roxb. & Shornolata & Parasite & Aug-Mar & Rare & Nusrat 22 \\
\hline \multicolumn{6}{|l|}{ Boraginaceae } \\
\hline Heliotropium indicum L. & Hatisur & Herb & Jan-Dec & Common & Nusrat 15 \\
\hline \multicolumn{6}{|l|}{ Verbenaceae } \\
\hline Clerodendrum viscosum Vent. & Vat & Shrub & Jan-Jul & Common & Nusrat 199 \\
\hline Lippia alba (Mill.) Briton et Wilson & Gondhapata & Shrub & Jan-Dec & Common & Nusrat 113 \\
\hline Tectona grandis L.f. & Shegun & Tree & Jul-Nov & Common & Nusrat 56 \\
\hline \multicolumn{6}{|l|}{ Lamiaceae } \\
\hline Anisomeles indica $\mathrm{L}$. & Gobura & Herb & Oct-Jun & Rare & Nusrat 181 \\
\hline Hyptis suaveolens Poit. & Bilatitulsi & Herb & Jan-Dec & Common & Nusrat 27 \\
\hline Leonurus sibricus Linn. & Roktodron & Herb & Jan-Dec & Rare & Nusrat 137 \\
\hline Leucas aspera (Willd.) Link & Dondokolos & Herb & Jan-Dec & Common & Nusrat 82 \\
\hline Mentha viridis $\mathrm{L}$. & Pudinapata & Herb & Jul-Jun & Common & Nusrat 84 \\
\hline Ocimum tenuiflorum $\mathrm{L}$. & Tulsi & Herb & Oct-Mar & Common & Nusrat 83 \\
\hline
\end{tabular}


Table 1 contd.

\begin{tabular}{|c|c|c|c|c|c|}
\hline Taxa & Bengali name & Habit & Phenology & $\begin{array}{l}\text { Status of } \\
\text { occurrence }\end{array}$ & $\begin{array}{l}\text { Voucher } \\
\text { number }\end{array}$ \\
\hline \multicolumn{6}{|l|}{ Oleaceae } \\
\hline Jasminum sambac (L.) Ait. & Beli & Shrub & Mar-Jul & Common & Nusrat 189 \\
\hline \multicolumn{6}{|l|}{ Scrophulariaceae } \\
\hline Scoparia dulcis $\mathrm{L}$. & Bandhony & Herb & Jan-Dec & Common & Nusrat 49 \\
\hline \multicolumn{6}{|l|}{ Acanthaceae } \\
\hline $\begin{array}{l}\text { Andrographis paniculata (Burm.f.) } \\
\text { Wall. }\end{array}$ & Kalomegh & Herb & Nov-May & Common & Nusrat 116 \\
\hline Justicia gendarussa Burm. f. & Jagatmadan & Shrub & Dec-May & Common & Nusrat 162 \\
\hline J. adhatoda $\mathrm{L}$. & Basok & Shrub & Jan-Apr & Common & Nusrat 03 \\
\hline Nelsonia canescens (Lamk.) Spreng. & Para-mul & Herb & Jan-Dec & Common & Nusrat 58 \\
\hline Phaulopsis imbricata (Forssk.) & Not known & Herb & Dec-Mar & Rare & Nusrat 04 \\
\hline \multicolumn{6}{|l|}{ Sweet } \\
\hline \multicolumn{6}{|l|}{ Pedaliaceae } \\
\hline Sesamum indicum $\mathrm{L}$. & Til & Herb & Feb-Oct & Common & Nusrat 190 \\
\hline \multicolumn{6}{|l|}{ Rubiaceae } \\
\hline Ixora coccinea $\mathrm{L}$. & Rangon & Shrub & Jan-Dec & Common & Nusrat 198 \\
\hline Morinda citrifolia $\mathrm{L}$. & Haldi kachu & Tree & May-Nov & Rare & Nusrat 104 \\
\hline $\begin{array}{l}\text { Neolamarckia cadamba (Roxb.) Merr. } \\
\text { Asteraceae }\end{array}$ & Kadom & Tree & Jul-Nov & Common & Nusrat 154 \\
\hline Ageratum conyzoides $\mathrm{L}$. & Fulkuri & Herb & Nov-Jun & Common & Nusrat 13 \\
\hline Blumea lacera (Burm.f.) DC & Barakukshima & Herb & Nov-Jul & Common & Nusrat 170 \\
\hline \multicolumn{6}{|l|}{ Robinson } \\
\hline Enhydra fluctuans Lour. & Helencha & Herb & Jan-Apr & Common & Nusrat 125 \\
\hline Mikania cordata (Burm. f.) & Taralota & Herb & Oct-Feb & Common & Nusrat 68 \\
\hline \multicolumn{6}{|l|}{ Robinson } \\
\hline Spilanthus calva DC. & Marhatatiga & Herb & Jan-Dec & Common & Nusrat 14 \\
\hline Synedrella nodiflora (L.) Gaertn. & Shialmoti & Herb & Jan-Dec & Common & Nusrat 126 \\
\hline \multicolumn{6}{|l|}{ LILIOPSIDA } \\
\hline \multicolumn{6}{|l|}{ Hydrocharitaceae } \\
\hline Ottelia alismoides (L.) Pers. & Panikola & Herb & June-Dec & Common & Nusrat 81 \\
\hline \multicolumn{6}{|l|}{ Arecaceae } \\
\hline Areca catechu $\mathrm{L}$. & Supari & Tree & Jan-Dec & Common & Nusrat 11 \\
\hline Borassus flabellifer $\mathrm{L}$. & Tal & Tree & Jan-Oct & Common & Nusrat 123 \\
\hline Calamus gracilis Roxb. & Raton & Tree & Apr-Oct & Rare & Nusrat 66 \\
\hline Cocos nucifera $\mathrm{L}$. & Narikel & Tree & Mar-Jul & Common & Nusrat 169 \\
\hline Phoenix sylvestris Roxb. & Khejur & Tree & Dec-Jul & Common & Nusrat 67 \\
\hline Alocasia macrorrhizos (L.) G. Don & Mankachu & Herb & Jul-Oct & Common & Nusrat 09 \\
\hline Amorphollus bulbifer $\mathrm{Bl}$. & Ul kachu & Herb & May-Oct & Rare & Nusrat 63 \\
\hline Colocasia esculenta Schott & Kochu & Herb & May-Oct & Common & Nusrat 122 \\
\hline Pistia stratiotes $\mathrm{L}$. & Topa-pana & Herb & Oct-Mar & Common & Nusrat 168 \\
\hline Typhonium trilobatum (L.) Schott. & Ghetkachu & Herb & Apr-Oct & Common & Nusrat 64 \\
\hline Xanthosoma violaceum Schott. & Dastorkachu & Herb & Apr-Oct & Common & Nusrat 10 \\
\hline
\end{tabular}


Table 1 contd.

\begin{tabular}{|c|c|c|c|c|c|}
\hline Taxa & Bengali name & Habit & Phenology & $\begin{array}{l}\text { Status of } \\
\text { occurrence }\end{array}$ & $\begin{array}{l}\text { Voucher } \\
\text { number }\end{array}$ \\
\hline \multicolumn{6}{|l|}{ Lemnaceae } \\
\hline Lemna purpusilla Torrey & Khudipana & Herb & Sep-Dec & Common & Nusrat 29 \\
\hline \multicolumn{6}{|l|}{ Commelinaceae } \\
\hline Commelina bengalensis $\mathrm{L}$. & Dholpata & Herb & Feb-Dec & Common & Nusrat 72 \\
\hline \multicolumn{6}{|l|}{ Cyperaceae } \\
\hline Cyperus compressus L. & Chanch & Herb & Jan-Dec & Rare & Nusrat 76 \\
\hline \multicolumn{6}{|l|}{ Poaceae } \\
\hline Brachiaria kurzii (Hook. f.) A. Camus & Not known & Herb & Jan-Dec & Rare & Nusrat 43 \\
\hline B. mutica Stapf & Para gash & Herb & Nov-Mar & Common & Nusrat 149 \\
\hline Chrysopogon aciculatus (Retz.) Trin. & Premkanta & Herb & Jan-Dec & Common & Nusrat 98 \\
\hline Cynodon dactylon (L.) Pers. & Durbaghas & Herb & Jul-Dec & Common & Nusrat 169 \\
\hline Imperata cylindrica (L.) P. Beauv & Ulookash & Shrub & Oct-Jan & Common & Nusrat 150 \\
\hline Isachne globosa (Thunb.) Kuntze & Not known & Herb & Jan-Dec & Rare & Nusrat 194 \\
\hline Oryza latifolia Desv. & Jangli dhan & Herb & Jul-Feb & Rare & Nusrat 99 \\
\hline O. sativa $\mathrm{L}$. & Dhan & Herb & Sep-Jun & Common & Nusrat 44 \\
\hline Panicum repens $\mathrm{L}$. & Dhanighas & Herb & Jan-Dec & Common & Nusrat 195 \\
\hline Paspalum scrobiculatum L. & Goicha & Herb & Jan-Dec & Common & Nusrat 46 \\
\hline Saccharum spontenum $\mathrm{L}$. & Kash & Herb & Jan-Dec & Common & Nusrat 100 \\
\hline Thysanolaena maxima (Roxb.) Kuntze & Jharu phul & Herb & Sep-Apr & Rare & Nusrat 151 \\
\hline \multicolumn{6}{|l|}{ Bromeliaceae } \\
\hline Ananas comosus (L.) Merr. & Anarosh & Herb & Feb-Jul & Common & Nusrat 17 \\
\hline \multicolumn{6}{|l|}{ Musaceae } \\
\hline Musa paradisiaca $\mathrm{L}$. & Kola & Herb & Jan-Dec & Common & Nusrat 146 \\
\hline \multicolumn{6}{|l|}{ Zingiberaceae } \\
\hline Curcuma longa $\mathrm{L}$. & Holud & Herb & Aug-Oct & Common & Nusrat 200 \\
\hline Zingiber officinale Rosc. & Ada & Herb & Sep-Nov & Common & Nusrat 161 \\
\hline \multicolumn{6}{|l|}{ Marantaceae } \\
\hline $\begin{array}{l}\text { Schumannianthus dichotomus (Roxb.) } \\
\text { Gagnep. }\end{array}$ & Patibet & Shrub & Dec-Mar & Rare & Nusrat 32 \\
\hline \multicolumn{6}{|l|}{ Pontederiaceae } \\
\hline Eichhornia crassipes (Mart.) Solms & Kachuripana & Herb & Jan-Dec & Common & Nusrat 45 \\
\hline Monochoria hastata (L.) Solms & Jolpana & Herb & Jan-Dec & Common & Nusrat 103 \\
\hline \multicolumn{6}{|l|}{ Liliaceae } \\
\hline Allium cepa $\mathrm{L}$. & Piyaj & Herb & Feb-Jun & Common & Nusrat 183 \\
\hline A. sativum $\mathrm{L}$. & Rosun & Herb & Feb-Apr & Common & Nusrat 139 \\
\hline Zephyranthes tubispatha $\mathrm{L}$. & Rain lily & Herb & Jun-Aug & Rare & Nusrat 86 \\
\hline
\end{tabular}

\section{Potential of the angiospermic flora}

\section{Medicinal Plants}

Potential of plant species of Gafargaon upazzila has been assessed and medicinal uses of the angiospermic flora by the local people have been recorded during field investigation. The study has identified 45 medicinal plants used for treatment of several diseases by the local people. The medicinal plant species with their part(s) used and uses are presented in Table 2. 
Table 2. Medicinal plants of Gafargaon upazila along with their part(s) used and diseases.

\begin{tabular}{|c|c|c|}
\hline Species & Part(s) used & Diseases \\
\hline Abroma augusta & Root & Urinary problem \& menstrual problems \\
\hline Achyranthus aspera & Root & Jaundice \\
\hline Adhatoda zeylanica & Leaf & Cold and cough \\
\hline Aegle marmelos & Fruit, root & Dysentery and diarrhoea \\
\hline Ageratum conyzoides & Leaf, stem, root & Fever, chronic ulcers and pneumonia \\
\hline Albizia procera & Leaf, bark & Insecticide and fish poisoning \\
\hline Alstonia scholaris & Bark & Asthma and fever \\
\hline Amaranthus spinosus & Leaf, root, stem & Rheumatism, blood purifier and irregular menstruation \\
\hline A. viridis & Whole plant & Snake-bite \\
\hline Annona reticulata & Bark & Diarrhoea \\
\hline Aphanamixis polystachya & Bark & Lever diseases and spleen \\
\hline Artocarpus heterophyllus & Root & Asthma and diarrhoea \\
\hline Averrhoa carambola & Fruit & Piles \\
\hline Blumea lacera & Root & Mouth diseases \\
\hline Cajanus cajan & Leaf & Diabetes and jaundice. \\
\hline Centella asiatica & Whole plant & Ulcer and dysentery \\
\hline Clerodendrum viscosum & Leaf, roots & Skin diseases, tumors and snake-bite \\
\hline Coccinia grandis & Leaf & Skin diseases and diabetes \\
\hline Colocasia esculenta & Leaf, corm & Astringent, scorpion bites and stimulant \\
\hline Commelina bengalensis & Whole plant & Urinary burning, sores and itches \\
\hline Cuscuta reflexa & Stem & Jaundice \\
\hline Cynodon dactylon & Whole plant & Stop bleeding and toothache. \\
\hline Dillenia indica & Fruit & Diarrhoea and dysentery \\
\hline Ficus hispida & Fruit & Diabetes \\
\hline F. religiosa & Bark & Toothache and skin diseases \\
\hline Glycosmis pentaphylla & Leaf, stem & Jaundice and toothache \\
\hline Heliotropium indicum & Leaf & Fever \\
\hline Holarrhena antidysenterica & Bark & Dysentery and elephantiasis \\
\hline Hyptis suaveolens & Leaf, seed & Stomachache \\
\hline Litsia glutinosa & Bark & Dysentery and diarrhoea \\
\hline Melia azedarach & Leaf & Small pox, fever and antiseptic \\
\hline Mikania cordata & Leaf & Wounds, itches and dyspepsia \\
\hline Mimosa pudica & Root & Jaundice, blood pressure and ulcer \\
\hline Moringa oleifera & Leaf, fruit, bark & Dysentery, vomiting, cold and cough and abscesses. \\
\hline Ocimum tenuiflorum & Leaf & Gastric disorder, cold, cough and ring worm \\
\hline Oxalis corniculata & Leaf & Antiscorbutic and antidote \\
\hline Peperomia pellucida & Leaf & Asthma \\
\hline Phoenix sylvestris & Fruit & Fever, Heart disease and abdominal complaints \\
\hline Piper nigrum & Fruit & Fever and bronchitis \\
\hline Rauvolfia serpentina & Root & Nervous agitation, high blood pressure and sound sleeping \\
\hline Scoparia dulcis & Whole plant & Kidney problems and diabetes \\
\hline Sida cordata & Fruit, flower & Burning complaints \\
\hline Solanum torvum & Root & Cough and toothache \\
\hline Spilanthus calva & Root & Toothache \\
\hline Syzygium cumini & Leaf, seed, bark & Diabetes, chronic diarrhoea and sore throats \\
\hline
\end{tabular}




\section{Economically and culturally important plant species}

The present study shows the role of angiospermic flora in the local communities. The local people rely on surrounding plant wealth not only for their health care, but also for food and other life accessories. Apart from medicinal uses several species are economically and culturally important. The species having economic and cultural importance are documented in Table 3.

Table 3. Plant species of Gafargaon upazila having economic and cultural importance.

\begin{tabular}{|c|c|c|}
\hline Species & Parts used & Economic and cultural importance \\
\hline Aegle marmelos & Fruit, leaf & $\begin{array}{l}\text { Fruits are edible. Leaves are used by Hindu community in } \\
\text { religious festival }\end{array}$ \\
\hline Alstonia scholaris & Wood & Used as furniture \\
\hline Annona squamosa & Fruit & Fruits are edible \\
\hline Artocarpus heterophyllus & Wood & Used as furniture \\
\hline Borassus flabellifer & Fruit, leaf & $\begin{array}{l}\text { Fruits are edible. Fibre is used in making mats, hats, brushes } \\
\text { and brooms }\end{array}$ \\
\hline Cocos nucifera & Fruit, leaf & $\begin{array}{l}\text { As drinks. Fibre is used in making brushes and brooms. Also } \\
\text { in religious festival fruits are used by the Hindu community }\end{array}$ \\
\hline Colocasia esculenta & Whole plant & Used as vegetables \\
\hline Curcuma longa & Rhizome & $\begin{array}{l}\text { The Hindu community uses the rhizome in their religious } \\
\text { festival }\end{array}$ \\
\hline Dillenia indica & Fruit & Fruits are used as vegetable, also used in making pickles \\
\hline Ficus banghalelsis & Leaf & $\begin{array}{l}\text { Leaves are employed by the Hindu people in their religious } \\
\text { festival }\end{array}$ \\
\hline F. hispida & Leaf & Leaves are used as vegetable \\
\hline F. religiosa & Leaf & $\begin{array}{l}\text { Leaves are employed by the Hindu people in their religious } \\
\text { festival }\end{array}$ \\
\hline Glycosmis pentaphylla & Twig & Young twigs are used as tooth-brush. \\
\hline Lawsonia inermis & Leaf & $\begin{array}{l}\text { Leaves are employed by the Hindu people in their religious } \\
\text { festival }\end{array}$ \\
\hline Mangifera indica & Leaf & $\begin{array}{l}\text { Fruits edible. Leaves are used by the Hindu people in their } \\
\text { religious festival }\end{array}$ \\
\hline Musa paradisiaca & Fruit, leaf & $\begin{array}{l}\text { Fruits edible. Leaves are employed by the Hindu people in } \\
\text { their religious festival }\end{array}$ \\
\hline Ocimum tenuiflorum & Whole plant & $\begin{array}{l}\text { In religious festival the Hindu community used this whole } \\
\text { plant }\end{array}$ \\
\hline Oxalis corniculata & Whole plant & Used as leafy vegetables \\
\hline Phoenix sylvestris & Fruit, leaf & $\begin{array}{l}\text { Fruits are edible. Fibre is used in making mats, hats, brushes } \\
\text { and brooms }\end{array}$ \\
\hline Solanum torvum & & Used as vegetables \\
\hline Syzgium cumini & Wood & Used for high class furniture \\
\hline
\end{tabular}

The present study revealed a number of threats based on the observations and group discussion with local people which might lead to cause angiospermic flora to diminish. Some of the important threats to the flora are habitat degradation, modern agriculture, urbanization, overexploitation of medicinal plants, lack of knowledge of collection technique, lack of awareness on biodiversity, and exotic plantation. Consequently, some species are extinct in the wild and many of them are at the verge of extinction. In order to save the plant resources from further annihilation urgent measures to be adopted for their conservation and sustainable uses including protection of 
habitats, public awareness on biodiversity conservation, and applying both ex-situ and in-situ conservation approaches for the medicinal and threatened species.

\section{Acknowledgement}

The financial support received from the Centre for Advanced Studies in Biological Sciences, University of Dhaka is gratefully acknowledged.

\section{References}

Ahmed, Z.U., Begum, Z.N.T. Hassan M.A., Khondker, M., Kabir, S.M.H., Ahmad, M., Ahmed, A.T.A., Rahman, A.K.A. and Haque, E.U. (Eds.) 2008-2009. Encyclopedia of Flora and Fauna of Bangladesh, Vols. 6-12. Asiatic Society of Bangladesh, Dhaka.

Alam, M.S., Hassan, M.A. and Uddin, M.Z. 2006. A preliminary checklist of the angiospermic flora of Ghagotia union under Kapasia Upazila in Gazipur district, Bangladesh. Bangladesh J. Plant Taxon. 13(2): 155-170.

Arefin, M.S., Hossain, M.K. and Hossain, M.A. 2017. Plant diversity of Sonadia island - An ecologically critical area of south-east Bangladesh. Bangladesh J. Plant Taxon. 24(1): 107-116.

BBS (Bangladesh Bureau of Statistics) 2018. Statistical Pocket Book Bangladesh 2017. Statistics and Informatics Division, Ministry of Planning, Government of the People's Republic of Bangladesh.

Bridson, D.M. and Forman, F. 1989. In: Bridson, D.M. and Forman, F. (Eds), The Herbarium Handbook. Royal Botanic Gardens, Kew, 214 pp.

Cronquist, A. 1981. An Integrated System of Classification of Flowering Plants. Columbia University Press, New York, 1262 pp.

Dassanayake, M.D and Fosberg, F.R. (Eds). 1980-1985. A Revised Handbook to the Flora of Ceylon, Vols. 16. Amerind Publishing Co. Pvt. Ltd., New Delhi.

Haque, A.K.M. Kamrul, Khan, S.A., Uddin, S.N. and Shetu, S.S. 2018. An annotated checklist of the angiospermic flora of Rajkandi Reserve Forest of Moulvibazar, Bangladesh. Bangladesh J. Plant Taxon. 25(2): 187-207.

Hooker, J.D. 1872-1897. The Flora of British India, Vols. 1-7. L. Reeve \& Co. Ltd., Kent, England.

Huq, A.M. 1986. Plant Names of Bangladesh. Bangladesh National Herbarium, BARC, Dhaka, Bangladesh, pp. 1-289.

Khan, M.S. (Ed.) 1972-1987 Flora of Bangladesh. Nos. 1-39. Bangladesh National Herbarium and Bangladesh Agricultural Research Council, Dhaka.

Khan, M.S. and Huq, A.M. 2001. The vascular flora of Chunati Wildlife Sanctuary in south Chittagong, Bangladesh. Bangladesh J. Plant Taxon.7(1): 47-64.

Khan, M.S. and Rahman, M.M. 1989-2002 (Eds.). Flora of Bangladesh. Fasc. 40-53. Bangladesh National Herbarium, Dhaka.

Moniruzzaman, M., Hassan, M.A., Rahman, M.M., Layla, S. and Islam, M.R. 2012. A preliminary checklist of the angiospermic flora of Daulatpur Upazila in Kushtia district, Bangladesh. J. Asiat. Soc. Bangladesh (Sci.) 38(1): 53-65.

Prain, D. 1903. (Indian Reprint 1981). Bengal Plants. Vols. 1 \& 2. Bishen Singh Mahendra Pal Singh Dehra Dun, India, $663 \mathrm{pp}$.

Rahman, M.O. and Alam, M.T. 2013. A taxonomic study on the angiospermic flora of Trishal Upazila, Mymensingh. Dhaka Univ. J. Biol. Sci. 22(1): 63-74.

Rahman, M.O., Antara, R.T., Begum, M. and Hassan, M.A. 2012. Floristic diversity of Dhamrai upazila of Dhaka, Bangladesh with emphasis on medicinal plants. Bangladesh J. Bot. 41(1): 71-85.

Rahman, M.O., Begum, M. and Ullah, M.W. 2013. Angiosperm flora of sadar upazila of Munshiganj district, Bangladesh. Bangladesh J. Plant Taxon. 20(2): 213-231. 
Rahman M.O., Hassan, S. and Begum, M. 2019. Floristic study in Lalpur upazila of Natore district, Bangladesh: Identification, distribution and economic potential. J. Asiat. Soc. Bangladesh (Sci.) 45(1): 71-91.

Rahman, M.S., Hossain, G.M., Khan, S.A. and Uddin, S.N. 2015. An annotated checklist of the vascular plants of Sundarban mangrove forest of Bangladesh. Bangladesh J. Plant Taxon. 22(1): 17-41.

Rashid, M.H., Islam, S. and Kashem, S.B. 2018. Floristic diversity (Magnoliids and Eudicots) of Baraiyadhala National Park, Chittagong, Bangladesh. Bangladesh J. Plant Taxon. 25(2): 273-288.

Singh, H.B. and Subramaniam, B. 2008. Field Manual on Herbarium Techniques. National Institute of Science Communication and Information Resources, pp. 1-297.

The Plant List, 2013. The Plant List, a working list of all plant species. Version $1.1<$ http://www. theplantlist.org/>. Accessed on 21 October 2018.

TROPICOS, 2017. Tropicos.org. <www.tropicos.org>. Missouri Botanical Garden, Saint Louis, Missouri, USA. Accessed on 21 October 2018.

Tutul, E., Uddin, M.Z., Rahman, M.O. and Hassan, M.A. 2010. Angiospermic flora of Runctia sal forest, Bangladesh-2. Magnoliopsida (Dicots). Bangladesh J. Plant Taxon. 17(1): 33-53.

(Manuscript received on 2 April, 2019; revised on 1 November, 2019) 